\title{
MODEL FOR SOLAR PROTON RISK ASSESSMENT
}

\author{
M.A. Xapsos ${ }^{1}$, C. Stauffer ${ }^{2}$, G.B. Gee ${ }^{2}$, J.L. Barth ${ }^{1}$, E.G. Stassinopoulos ${ }^{1}$ and R.E. McGuire ${ }^{1}$ \\ ${ }^{1}$ NASA Goddard Space Flight Center, Greenbelt, MD 20771 USA \\ ${ }^{2}$ SGT Inc., Greenbelt, MD 20770 USA
}

\begin{abstract}
A statistical model for cumulative solar proton event fluences during space missions is presented that covers both the solar minimum and solar maximum phases of the solar cycle. It is based on data from the IMP and GOES series of satellites that is integrated together to allow the best features of each data set to be taken advantage of. This allows fluence-energy spectra to be extended out to energies of $327 \mathrm{MeV}$.
\end{abstract}

\section{INTRODUCTION}

The effects that solar particle events have on microelectronics, detectors and solar arrays are important considerations for many space missions. Of particular significance are those missions planned for geosynchronous orbits, polar orbits and for interplanetary missions as in NASA's new space initiative. Protons are by far the predominate particle type in solar particle events so it is therefore essential to have accurate models for spacecraft reliability and astronaut protection.

Designers and mission planners are required to assess the performance of microelectronic systems under a variety of conditions. One interest is the accumulation of solar proton event fluence over the duration of a mission. Confidence level approaches are often used so that riskcost-performance tradeoffs can be evaluated. Several useful models have previously been presented for estimating cumulative fluences during the solar maximum time period. The first such model was based on King's analysis of $>10$ to $>100 \mathrm{MeV}$ proton data during solar cycle 20 [1,2]. The Jet Propulsion Laboratory 1991 (JPL91) model has also been widely used in the radiation effects community $[3,4]$. It is based on data from solar cycles 20,21 and part of 22 , and covers the energy range of $>1$ to $>60 \mathrm{MeV}$. More recently, the Emission of Solar Protons (ESP) model has become available [5,6], utilizing data from 3 complete solar cycles, 20-22. The energy range of the true statistical model is $>1$ to $>100 \mathrm{MeV}$, and an extrapolation is used to reach $>300 \mathrm{MeV}$.

All of the above models cover the solar maximum time period and assume that the proton fluence contribution during solar minimum can be neglected. However, for missions that are planned largely or entirely for solar minimum there are no generally accepted guidelines. It would be useful to have environment guidelines available for this situation, considering the current reliance on commercial-off-the-shelf (COTS) microelectronics. In addition, there is interest in solar proton energies beyond $100 \mathrm{MeV}$ for several reasons. First, single event effects cross sections generally increase with increasing proton energy. Second, there can be significant shielding depths in modern spacecraft. The range of a $100 \mathrm{MeV}$ proton in aluminum is $36.8 \mathrm{~mm}$ and the range of a $327 \mathrm{MeV}$ proton is $278.8 \mathrm{~mm}$ so the shielding depth that can be accounted for increases by a factor of 7.6 when using these new model results. Third, high proton energies are significant for astronaut exposures and must be accounted for in the planning for manned missions. Finally, high energy protons contribute dose to aircrew. It should be noted, though, that ground level neutron monitor data must also be considered for the latter situation [7]. 


\section{CONSTRUCTION OF AN INTEGRATED SOLAR PROTON EVENT DATA BASE}

Previous models aimed at radiation effects applications have generally treated data from different instruments independently. For cxample, in our ESP model, we have used data from the Interplanetary Monitoring Platform (IMP) series of satellites for solar cycles 20 and 21 and switched over to the Geostationary Operational Environmental Satellites (GOES) data for cycle $22[5,6]$. However, the strengths and weaknesses of the measurements and available data from these instruments are to a large degree complementary. The Goddard Medium Energy (GME) instrument on IMP-8 has been operating nearly continuously since 1973 in an approximately circular orbit at 35 Earth radii. Thus, it is well positioned to measure the interplanetary particle fluxes. The proton differential flux data is provided in 30 energy bins ranging from 0.88 to 485 $\mathrm{MeV}$ [8], thus providing a broad energy range with sufficiently fine increments for well-defined and detailed energy spectra. The highest energy bin starts at $327 \mathrm{MeV}$ and defines the upper proton energy limit of this model. In addition, the detectors have low noise levels that are necessary for measuring the small high-energy fluences present in some events. The main disadvantage of this instrument is that the detectors saturate during some of the largest events. On the other hand, the GOES series of satellites have been operational in geostationary orbits since 1986. The available proton data comes in 10 energy bins ranging from 0.6 to $700 \mathrm{MeV}$, although the detector noise levels in the high-energy range are rather high. An important strength of the GOES instruments is that they perform very well during high flux rates.

We have undertaken an extensive new analysis of the IMP and GOES solar proton data aimed at combining the best data features from different instruments into an Integrated Data Set (IDS). Based on a number of factors, the GME data have been used as a starting point for the absolute values of measured proton fluxes. First, the orbit of the IMP-8 satellite is more appropriate for measuring interplanetary fluxes. Second, the data are available in finer energy increments. Third, there are noticeable differences in measured fluxes by instruments on different GOES satellites during the same event. (See Fig.2, for example.) It is unknown whether this is due to magnetospheric effects, different instruments or a combination of both. Finally, there is some uncertainty in the geometrical factors of the GOES instrumentation [7]. Thus, the GOES data have been used to fill in gaps in the GME data such as during times of very high fluxes when the GME instrument is saturated. This is illustrated in Fig. 1. for the well known October and November 1989 time period. In this case the GOES-7 Space Environment Monitor (SEM) data from a similar energy range have been scaled to fit the flux vs. time profile measured by the GME instrument at a time when the data are reliable. The GME data are shown by circles and the scaled SEM data are shown by X's. It is seen that the resulting time profile that is achieved is reasonable. The result of this procedure, in terms of the event fluence-energy spectrum is shown in Fig. 2 compared to the GOES-6 and -7 measurements. Again there is reasonable agreement. The net result is that the IDS achieves reasonable flux vs. time profiles and integrated fluences that are consistent with both instrument measurements. In addition, the energy dependence of fluences and fluxes can be studied in greater detail.

The basic procedure outlined above using the GME and GOES instruments was followed for data from time periods ranging from 1986 to 2001, beyond which the GME data are not currently available. For time periods ranging from 1973 to 1986 , the GME data were similarly supplemented with data from the Charged Particle Measurement Experiment (CPME) onboard IMP-8. Data from the analysis of King has also been included in our model, which is from the solar maximum phase of cycle 20 [1]. This covers the time period from 1966 to 1972. These 
data are from the IMP-3, -4 and -5 satellites and cover the energy range of $>10$ to $>100 \mathrm{MeV}$. In this analysis, King used all available proton flux measurements. Comparison of four instrument data sets from the IMP-4 satellite showed agreement to within $25 \%$. The results of the current model are not significantly changed if these cycle 20 datia are exciuded from our analysis. However, we choose to include it so that we have a reasonably complete long-term data base of solar proton measurements made on satellites.

Due to the inclusion of the solar minimum time period in our new model, it was necessary to analyze smaller event sizes than has been done previously. A total of 503 events have been included in our data base for this work, 77 of which occurred during solar minimum. In order to include small event fluences in both the low and high energy portions of the spectra, an event was analyzed for this model if either the peak differential flux in the 1.15 to $1.43 \mathrm{MeV}$ channel exceeded $4 \mathrm{~cm}^{-2} \mathrm{~s}^{-1} \mathrm{sr}^{-1} \mathrm{MeV}^{-1}$ or the same quantity in the 42.9 to $51.0 \mathrm{MeV}$ channel exceeded $0.001 \mathrm{~cm}^{-2} \mathrm{~s}^{-1} \mathrm{sr}^{-1} \mathrm{MeV}^{-1}$. Individual background subtractions were carried out for all channels of the 503 events considered.

\section{RESULTS}

It is well known that solar particle event frequencies and magnitudes vary throughout the solar cycle. Previous statistical analysis has shown that these properties are well approximated to be bimodal in character, and can be separated into solar maximum and solar minimum time periods [4]. Following the approach of Feynman, it is assumed that the solar maximum period is 7 years in duration, beginning 2.5 years before and ending 4.5 years after the date of the peak sunspot number [9]. This date for solar cycles $20-23$ is taken as 1968.9, 1979.9, 1989.9 and 2000.2 , respectively. The remaining portion of each solar cycle is considered to be solar minimum.

\section{A. Model for Solar Minimum}

Motivation for the solar minimum model comes from the observation that indicators of solar activity such as sunspot numbers and solar $10.7 \mathrm{~cm}$ radio flux tend to a low and fairly constant value during solar minimum time periods [10], even though they vary substantially from one solar maximum period to the next. Since we know that solar proton fluxes are comparatively low during solar minimum $[9,11]$, the assumption is made that there is a constant low level solar proton "background" flux present at all times during the solar cycle. During solar minimum the "background" flux represents a convenient average value for solar protons. In reality, though, flux increases occur in small bursts but our assumption of a constant value is sufficient for radiation effects applications. During solar maximum, high activity leads to high flux rates that are superimposed on this "background" level so that it becomes comparatively insignificant.

For the solar minimum model, we have thus analyzed these time periods to obtain 3 flux levels that can be used to represent this "background" level. (These levels should not be confused with galactic cosmic ray and inherent background noise, which has been subtracted out on an individual event basis.) This allows varying degrees of conservatism to be used. The flux levels are shown in Fig.3. First, there is the average solar proton flux vs. energy spectrum over all 3 solar minimum periods that occurred between 1966 and 2001. This level, shown by the triangles in Fig.3, is obtained by summing all solar minimum fluences and dividing by the total 
solar minimum time. A more conservative choice shown by the open squares is the "worst solar minimum period". This was obtained by summing the fluences from the solar minimum period at the end of cycle 20 and beginning of cycle 21 and dividing by the total time. This turns out to be the highest solar proton flux rate for the 3 solar nimimum periods in the data. The most conservative choice is the "worst solar minimum year", and is shown by the circles in the Figure. This is the one-year period beginning April 23, 1985 and ending April 22, 1986. The model calculates the predicted solar proton fluence-energy spectrum during solar minimum as the selected flux level times the mission time duration of interest.

Also shown in Fig. 3 for comparison purposes is the average solar proton flux per solar maximum year during the time period 1966 to 2001. These values are indicated by the squares. Comparing this to the average flux per solar minimum year, it is seen that the solar maximum values exceed the solar minimum values at $>1 \mathrm{MeV}$ by about an order of magnitude. At $>300$ $\mathrm{MeV}$ it is about 2 orders of magnitude. It can be concluded that the energy spectra, on average, are harder during solar maximum. This also makes sense physically because the sun is in a more highly disturbed state during solar maximum, generally resulting in greater energy release processes.

\section{B. Model for Solar Maximum}

The mathematical model of cumulative solar proton fluence for a mission has been described previously for solar maximum [5,6]. Several techniques were used including the Maximum Entropy Principle, data simulation using the measured event distribution, and Bootstrap-like methods to arrive at the conclusion that the probability distribution for cumulative fluence is well described as one that is lognormal. The parameters for a 1-year cumulative distribution are obtained directly from plotting the annual solar proton event fluences for each solar maximum year on lognormal probability paper. The distribution parameters for longer (or shorter) periods of time are then simply related to the 1-year parameters due to the Poissonian nature of events. Fig. 4 shows a typical comparison of the new results to other models. In this case it is for the $90 \%$ confidence level and for a mission length of 2 solar maximum years. Results for the King model and JPL91 model were obtained from the SPace ENVironment Information System (SPENVIS) web site [12], which has also implemented the ESP model. The spectral shape for the King model is based on the well-known August 1972 event and is therefore somewhat different than the other model results. In all cases, the energy range shown corresponds to the data range on which the model is based, i.e., no extrapolations are shown. Thus, the model differences seen in the figure are an indicator of model uncertainties. The JPL91 model, ESP model and this work all agree reasonably well from energies ranging from $>1$ to $>60 \mathrm{MeV}$. The largest differences occur for low energies but are within the uncertainties of the underlying data. The low energy measurements may well have the largest associated uncertainties due to failed anti-coincidence shields in the low energy GME and CPME detectors. In addition, the low energy GOES detectors probably measure slightly bigher fluxes than that seen in interplanetary space due to the presence of a small amount of trapped or quasi-trapped protons at geosynchronous altitudes. There is also a time-varying cutoff rigidity effect that can make the data difficult to interpret. In the $>60$ to $>100 \mathrm{MeV}$ range, the ESP Model and this work are in excellent agreement. The current work significantly extends the energy range of the statistical model out to $>327 \mathrm{MeV}$. This is more than 3 times the energy range of the ESP statistical model and more than 5 times the energy range of the JPL91 Model. 


\section{Cumulative Fluence for a Mission}

The total fluence accumulated over the course of a mission at a given confidence level is the sum of the solar maximum and solar minimum contributions. An example of such results is shown in Fig. 5 for 4 years of solar minimum followed by 7 years of solar maximum. The cumulative fluence for $>4.94,>28.7,>107$ and $>327 \mathrm{MeV}$ protons is plotted as a function of time. In this case the solar minimum fluences result from the application of the "worst solar minimum period" shown in Fig. 3. The solar maximum results are for a 7 year period at the $90 \%$ confidence level. It is seen that previous assumptions that solar minimum fluences are small compared to solar maximum fluences are justified. However, note that these differences are smaller for proton energies around a few MeV. For missions that occur during the solar minimum period, these new results may help relax design margins. However, it is important to note that the relaxation of margins can be limited by galactic cosmic ray proton fluxes, which increase during solar minimum.

\section{SUMMARY AND CONCLUSIONS}

In this work an extensive solar proton event data base was developed based on the IDS approach. This was used to significantly extend the energy range of our probabilistic model out to $>327 \mathrm{MeV}$. In addition, a description of the solar minimum time period was developed, thus providing the complete solar cycle dependence. Results support previous assumptions that solar particle event data can be described as bimodal in nature corresponding to the solar maximum and solar minimum time periods. Generally during solar minimum, the event frequencies are smaller, the event magnitudes are smaller, and the energy spectra are softer.

\section{REFERENCES}

[1] J.H. King, "Solar proton fluences for 1977-1983 space missions," J. Spacecraft, vol. 11, 401408 (1974)

[2] E.G. Stassinopoulos and J.H. King, "Empirical solar proton model for orbiting spacecraft applications," IEEE Trans. Aerospace and Elect. Sys., vol. 10, 442-450 (1974).

[3] J. Feynman, G. Spitale, J. Wang and S. Gabriel, "Interplanetary fluence model: JPL 1991," J. Geophys. Res., vol. 98, 13281-13294 (1993).

[4] J. Feynman, A. Ruzmaikin and V. Berdichevsky, "The JPL proton fluence model: an update," J. Atmospheric and Solar-Terrestrial Phys., vol. 64, 1679-1686 (2002).

[5] M.A. Xapsos, J.L. Barth, E. G. Stassinopoulos, E.A. Burke and G.B. Gee, "Model for emission of solar protons (ESP) - cumulative and worst-case event fluences," NASA Report TP-1999-209763, Marshall Space Flight Center, Alabama, Dec. 1999.

[6] M.A. Xapsos, G.P. Summers, J.L. Barth, E.G. Stassinopoulos and E.A. Burke, "Probability model for cumulative solar proton event fluences," IEEE Trans. Nucl. Sci. vol. 47, no. 3, 486-490 (June 2000). 
[7] C.S. Dyer, F. Lei, S.N. Clucas, D.F. Smart and M.A. Shea, "Solar particle enhancements of single-event effect rates at aircraft altitudes," IEEE Trans. Nucl. Sci., vol. 50, 2038-2045 (Dec. 2003).

[8] htp/spdf gsfc.nasagov/mp8_GME/GME_home htini, and references therein.

[9] J. Feynman, T.P. Armstrong, L. Dao-Gibner and S.M. Silverman, "New interplanetary fluence model," J. Spacecraft, vol. 27, 403-410 (1990).

[10] M.A. Xapsos, S.L. Huston, J.L. Barth and E.G. Stassinopoulos, "Probabilistic model for low altitude trapped proton fluxes," IEEE Trans. Nucl. Sci., vol. 49, 2776-2781 (Dec. 2002).

[11] J.L. Barth, NSREC Short Course, Snowmass Village, CO, July 1997.

[12] http://www spenvis.oma.be/spenvis.

\section{ACKNOWLEDGMENTS}

This work was supported by the NASA Space Environments and Effects Program and by the NASA R\&TD Core Capability Development Program.

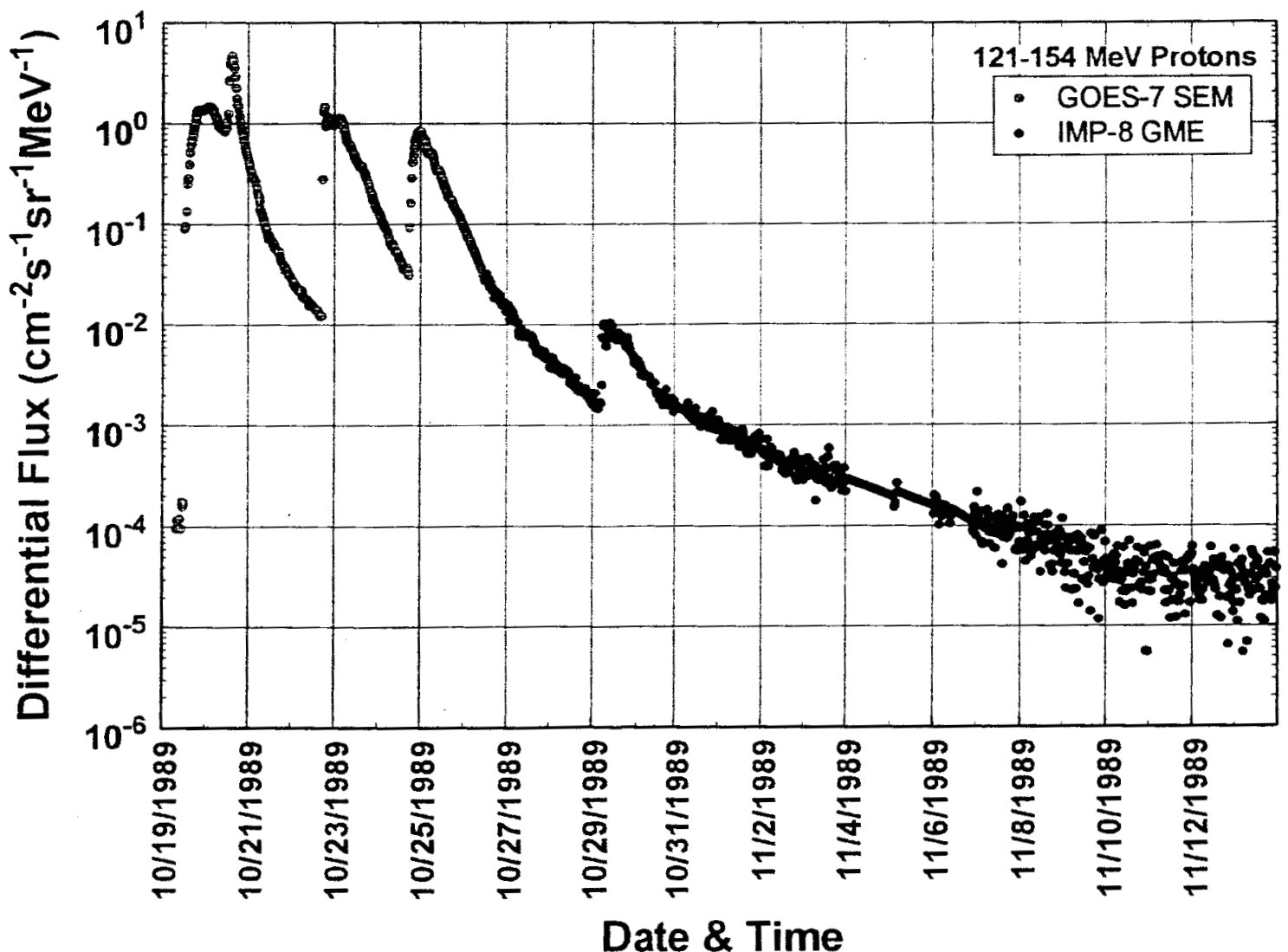

Fig.1. October and November 1989 differential fluxes obtained with the IDS for 121-154 MeV protons. 


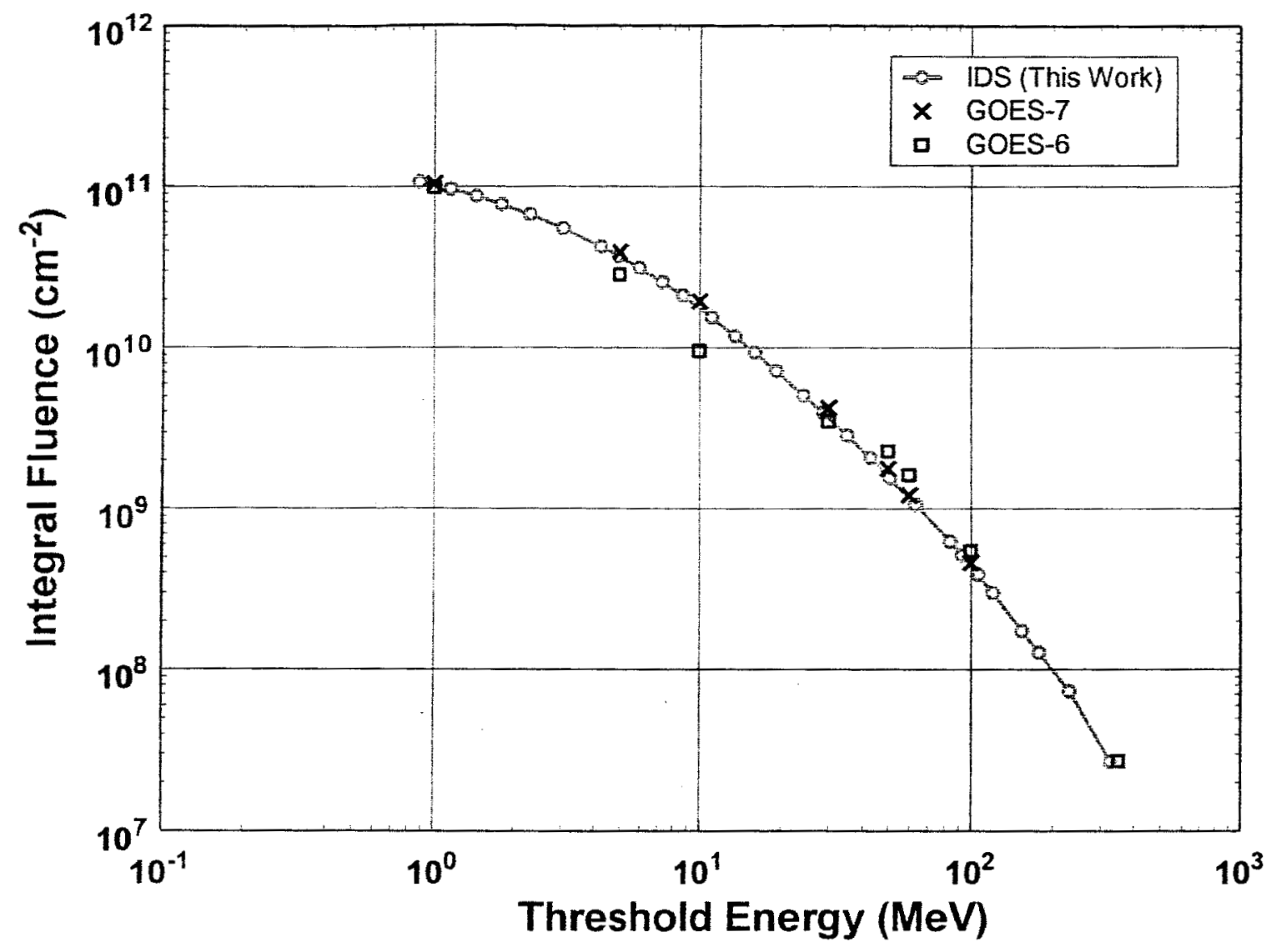

Fig.2. Integral fluence vs. energy spectra for the October and November 1989 time period. Results from the GOES6 and GOES-7 instrumentation are compared to the IDS approach. 


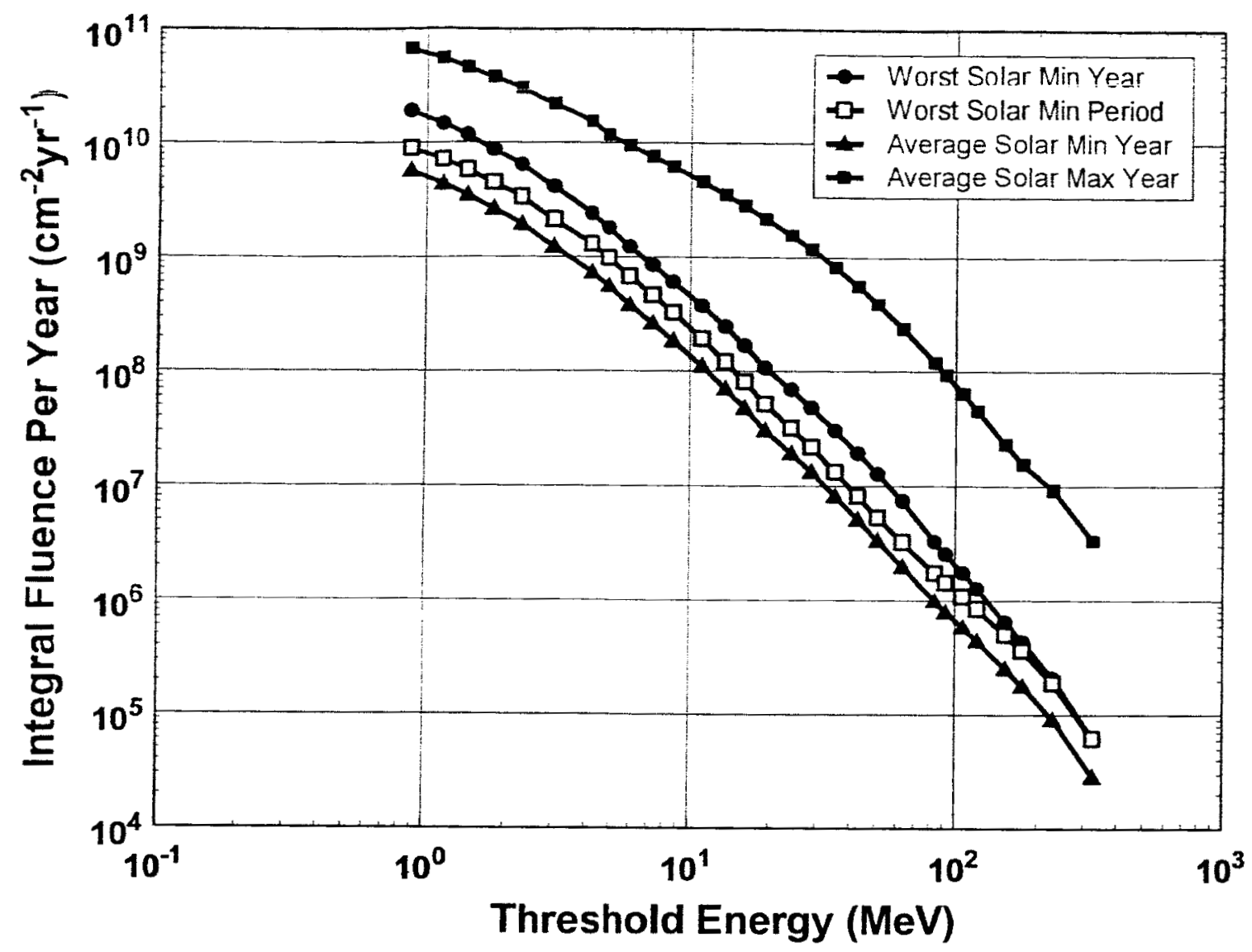

Fig.3. Proton flux vs. energy spectra for the 3 solar minimum model spectra. Also shown for comparison purposes is the average proton flux during solar maximum. 


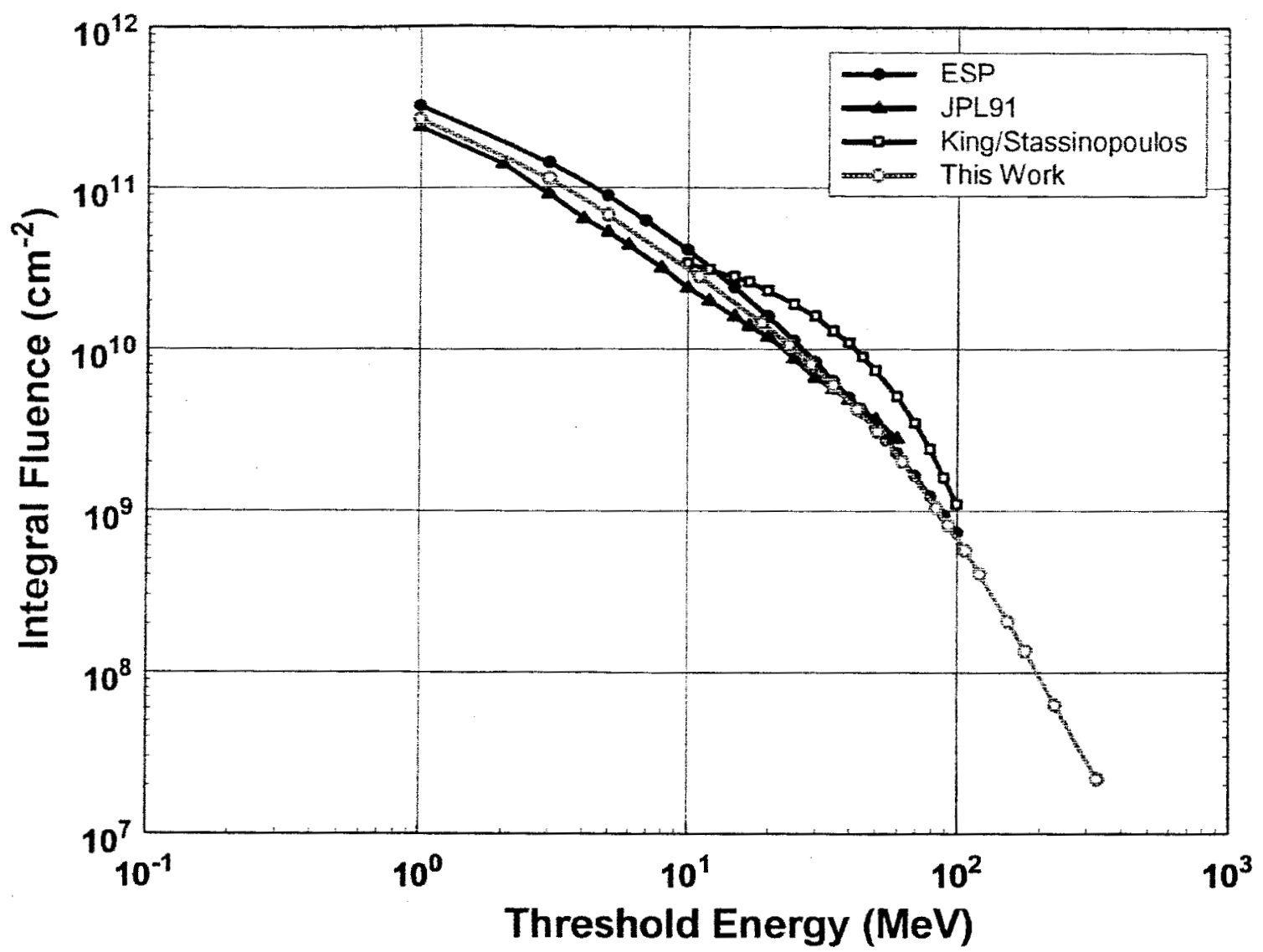

Fig.4. Comparison of the King/Stassinoponlos model, JPL91 model, ESP model and results of this work for 2 years during solar maximum at the $90 \%$ confidence level. 


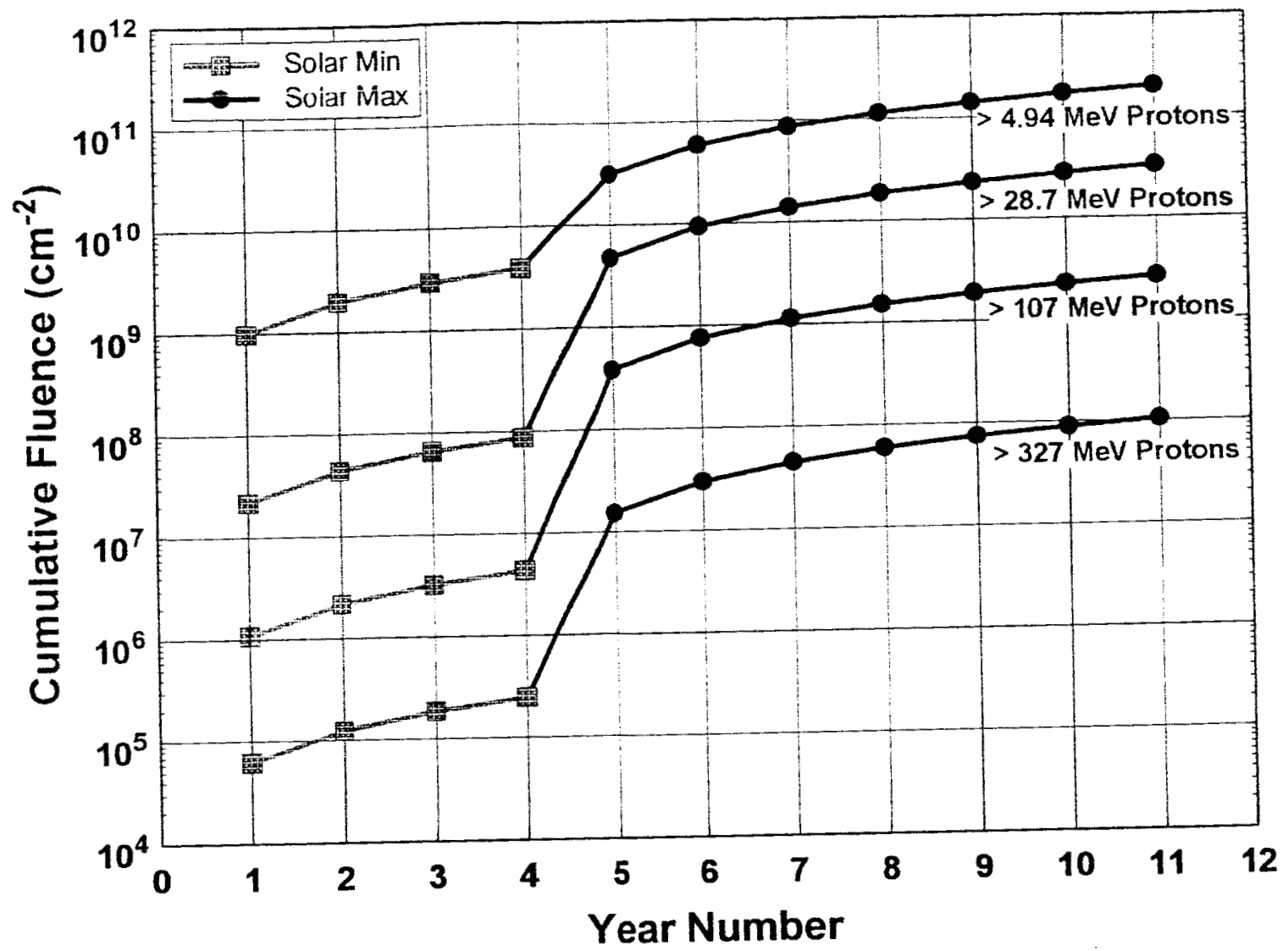

Fig.5. Cumulative fiuence for protons of different energy ranges over the course of an 11 year mission. The first 4 years are during solar minimum and the last 7 are during solar maximum. See text. 